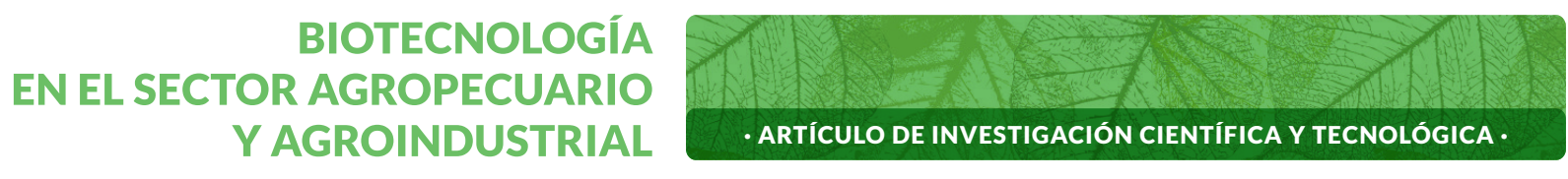

Vol. 19 No 1 · Enero - Junio 2021 · ISSN - 1692-3561 · ISSN-e 1909-9959 · DOI: https://doi.org/10.18684/BSAA(19)256-267

\title{
Los sistemas agroforestales y la incidencia sobre el estatus hídrico en árboles de cacao*
}

\section{Agroforestry systems and the incidence on water status of cacao trees}

\section{Sistemas agroflorestais e a incidência no estado hídrico em cacaueiros}

ORDOÑEZ-ESPINOSA, CLAUDIA-MERCEDES'; SUÁREZ-SALAZAR, JUAN-CARLOS; RANGEL-CHURIO, JESUS-ORLANDO ${ }^{3}$, SAAVEDRA-MORA, DAVID ${ }^{4}$

Historial del Artículo

Recibido para evaluación: 14 de Junio 2020.

Aprobado para publicación: 20 de Octubre 2020

* Proyecto de investigación de origen: "Composición florística, estructura y servicios ecosistémicos en sistemas agroforestales con Theobroma cacao L. en el departamento del Huila". Financiación: Servicio Nacional de Aprendizaje - SENA, Sistema de Investigación, Desarrollo Tecnológico e Innovación - SENNOVA, Centro de Formación Agroindustrial Regional Huila. Culminación: 30 de diciembre de 2018.

1 Servicio Nacional de Aprendizaje, Regional Huila, Centro de Formación Agroindustrial, Grupo de Investigación Agroindustrial. M Sc. Sistemas Sostenibles de Producción. Campoalegre, Colombia. https://orcid.org/0000-0003-1095-4468

2 Universidad de la Amazonía, Facultad de Ingeniería, Programa de Ingeniería Agroecológica, Maestría en Sistemas Sostenibles de producción, Centro de Investigaciones Amazónicas CIMAZ Macagual Cesar Augusto Estrada Gonzales, Grupo de Investigaciones Agroecosistemas y Conservación en Bosques Amazónicos- GAIA. PhD en Ciencias Biológicas. Florencia, Colombia. http://orcid.org/0000-0001-5928-1837

3 Universidad Nacional de Colombia, Facultad de Ciencias, Instituto de Ciencias Naturales, Grupo de Investigación en Biodiversidad y conservación PhD. en Biología. Bogotá, Colombia. https://orcid.org/0000-0002-7907-9393

4 Servicio Nacional de Aprendizaje, Regional Huila, Centro de Formación Agroindustrial, Grupo de Investigación Agroindustrial. M Sc. Agroforestería. Campoalegre, Colombia. https://orcid.org/0000-0002-2730-8545

Correspondencia: cordoneze@sena.edu.co 


\section{RESUMEN}

El cacao se cultiva bajo sistemas de producción como los agroforestales y libre exposición solar, su uso depende de las regiones agroecológicas donde se cultiva. El manejo del dosel de sombra incide en las condiciones microclimáticas que beneficia el comportamiento hídrico (flujo de savia $V_{s}$ y potencial hídrico $\Psi)$ de la planta de cacao. Se evaluó como la modificación del dosel de sombra (sistemas a libre exposición solar SLE y agroforestales con media $M_{P A R} y$ baja $B_{P A R}$ radiación transmitida) afectan el comportamiento hídrico en árboles de cacao en épocas contrastantes de precipitación. Para medir el $V_{s}$ y $\psi$ se instalaron sensores de calor y psicrómetros de tallo en árboles de cacao tipo CCN51. Se encontró un efecto del sistema de producción y época de monitoreo sobre el $V_{s} y \psi$ en las plantas de cacao. El comportamiento del $\psi$ fue mayor en SLE en las dos épocas, pero se acentuó más en la época de mínima precipitación. Se encontró un comportamiento contrario en $V_{s}$, donde esta variable fue mayor en $B_{P A R}$ durante la época de mínima precipitación. De acuerdo con los resultados obtenidos el manejo de doseles de sombra en cultivos de cacao bajo climas subóptimos incide positivamente en el estatus hídrico.

\section{ABSTRACT}

Cocoa is grown under production systems such as agroforestry and full sunlight exposition, its use depends on the agroecological regions where it is cultivated. The management of the shade canopy affects the microclimatic conditions that mainly benefit the hydric behaviour (sap flow $V_{s}$ and water potential $\Psi$ ) in the cocoa plant. It was evaluated how the modification of the shade canopy (full sunlight exposition systems SLE and agroforestry with $M_{P A R}$ medium and low $B_{P A R}$ transmitted radiation) affects the water status of cocoa trees in contrasting periods of precipitation. For the measurement of $V_{s}$ and $\psi$ heat sensors and stem psychrometers were installed in cocoa type CCN51 trees. An effect of the production system and monitoring season on $V_{s}$ and $\psi$ was found in cocoa plants. The $\psi$ was higher in SLE in both seasons, but it was more accentuated in the period of minimum precipitation. A contrary behavior was found in $V_{s}$, since in minimum precipitation this variable was greater in $B_{P A R}$. According to the results obtained, the management of shade canopies in cocoa crops under suboptimal climates positively affects water status.

\section{RESUMO}

Ocacau é cultivado sob diferentes sistemas de produção, como agroflorestais e exposição ao pleno sol, seu uso dependem das regiões agroecológicas onde é cultivada. O manejo do dossel de sombra tem um impacto nas condições microclimáticas

Cómo citar este artículo: ORDOÑEZ-ESPINOSA, CLAUDIA-MERCEDES; SUÁREZSALAZAR, JUAN-CARLOS; RANGEL-CHURIO, JESUS-ORLANDO, SAAVEDRAMORA, DAVID. Los sistemas agroforestales y la incidencia sobre el estatus hídrico en árboles de cacao. Biotecnología en el sector agropecuario y agroindustrial, v. 19, n. 1 , 2021, p. 256-267. Doi: https://doi.org/10.18684/BSAA(19)256-267

\section{PALABRAS CLAVE:}

Flujo de savia; Potencial hídrico; Disponibilidad de luz; Cobertura del dosel; cacao; agroforestales; hídrico.

\section{KEYWORDS:}

Sap flow; Water potential; Light availability; Canopy cover; cacao; Agroforestry; water.

\section{PALAVRAS-CHAVE:}

Fluxo de seiva; Potencial hídrico; Disponibilidade de luz; Cobertura do dossel; cacaueiros; agroflorestais; hídrico. 
que beneficiam principalmente o comportamento hídrico (fluxo de seiva $V_{s}$ e potencial hídrico $\psi$ ) da planta do cacaueiro. Foi avaliado como a modificação do dossel de sombra (sistemas de exposição ao pleno sol SLE e agroflorestais com médio $M_{P A R} e$ baixa $B_{\text {PAR }}$ radiação transmitida) afeta o comportamento hídrico do cacaueiro em períodos contrastantes de precipitação. Para

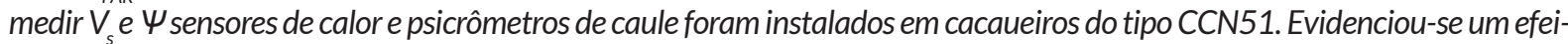
to do sistema de produção e do período de monitoramento em $V_{s}$ e $\Psi$ foi encontrado em plantas de cacau. $O \Psi$ foi maior no SLE nos dois períodos, mas foi mais acentuado no período de precipitação mínima. No entanto, um comportamento contrário foi encontrado em Vs, pois na precipitação mínima essa variável foi maior no $B_{P A R^{*}}$. De acordo com os resultados obtidos, o manejo de copa de sombra em cacau em climas subótimos tem um impacto positivo no status hídrico.

\section{INTRODUCCIÓN}

El cacao se cultiva en el mundo en sitios en los cuales la precipitación varía desde 1200 hasta 4000 mm anuales $[1,2,3]$ y la temperatura entre 21 y $32^{\circ} \mathrm{C}$ con un desempeño óptimo a nivel productivo [4]. Cuando el cultivo de cacao está expuesto a periodos prolongados de sequía se afectan de manera inmediata sus procesos fisiológicos (apertura de estomas, reducción de la fotosíntesis, metabolismo y respiración), como a largo plazo en procesos que indicen en la reducción de su crecimiento y rendimiento [5, 6, 7].

Las áreas con plantaciones de cacao en el departamento del Huila están expuestas a las variaciones climáticas producto del cambio climático [8, 9] debido a períodos prolongados de eventos globales como El Niño y La Niña [10], que aumenta la temperatura entre $1,4-4,8^{\circ} \mathrm{C}[11]$, generan cambios en los patrones de lluvias y regímenes de precipitación [12], y reducen la productividad [13]. La sensibilidad de las plantas de cacao al déficit hídrico se debe principalmente a características morfo lógicas de sus hojas, al presentar variaciones de area foliar que están relacionadas con la transpiración [14]; los vasos del xilema tienen mayor diámetro comparado con aquellas plantas que se encuentran en un estatus hídrico adecuado, y debido a este estrés las raíces se concentran entre 0,4-0,8 m de profundidad, que limita la absorción de agua en el suelo [15] y pueden aumentar la sensibilidad del cacao a la cavitación en periodos de sequía [16]. Si el aporte hídrico del suelo está por debajo del requerimiento hídrico estimado del árbol (1400-2000 mm/año) se verá afectado el proceso de floración (reducción en la tasa de producción de flores y el cuajado) [11], que influyen en el rendimiento del grano [13].

Actualmente en Colombia el cacao es cultivado en cuatro regiones agroecológicas: departamentos de Santander y Norte de Santander (altitudes entre 300 y 1200 msnm, precipitación entre 1500 y 2500 mm/año, temperatura entre 23 y $28^{\circ} \mathrm{C}$ ), departamentos del eje cafetero, Caldas, Quindío, Risaralda, Antioquia, Santander, Norte del Tolima, Boyacá (altitudes entre 500 y 1200 msnm, precipitación entre 1500 y 2000 mm/ año, temperatura entre 23 y $26^{\circ} \mathrm{C}$ ), Arauca, Meta, Urabá y Bajo Cauca (altitudes entre 0 y $500 \mathrm{msnm}$, precipitación > $2500 \mathrm{~mm} /$ año, temperatura entre 26 y $30^{\circ} \mathrm{C}$ ), Huila, Cauca, Valle del Cauca, Tolima, Costa Atlántica (altitudes entre 0 y 500 $\mathrm{msnm}$, precipitación $<1500 \mathrm{~mm} /$ año, temperatura entre 26 y $30^{\circ} \mathrm{C}$ ), pero estas regiones presentan condiciones de precipitación contrastantes (<1200 mm hasta $4000 \mathrm{~mm}$ anuales) [17].

Algunas de estas regiones no presentan restricciones en clima y suelo que afecten el rendimiento del grano de manera importante (suelo profundos $>100 \mathrm{~cm}$, bien drenados, temperatura entre 24 y $28^{\circ} \mathrm{C}$ y precipitación anual entre 1800 y 2600 mm/año que garantizan las necesidades hídricas del cultivo), pero cultivos de cacao ubicados en Valles interandinos secos presentan restricciones en disponibilidad de agua con precipitaciones anuales < $1200 \mathrm{~mm}$ que pueden ser no aptas para la producción de cacao [13].

En el departamento del Huila la actividad cacaotera se concentra en 10 municipios, con mayor producción en Campoalegre, Rivera, Algeciras y Hobo. Esta región se caracteriza por condiciones de bosque seco tropical, con precipitaciones que varían entre $668 \mathrm{~mm}$ y $1500 \mathrm{~mm}$ anuales, una marcada estacionalidad de lluvias de una época seca larga (Julio, Agosto y Septiembre) y seca corta (Enero y Febrero), alta radiación solar mensual (1498,2 micromol $\mathrm{m}^{2} / \mathrm{s}$ ) y brillo solar ( 6 horas de sol/día) que pueden incidir en el uso de agua por las plantas de cacao, en respuesta a las condiciones ambientales [18]. 
Estas fluctuaciones del clima han motivado a los productores a implementar diferentes arreglos agroforestales con el cultivo de cacao $[19,20]$, como una estrategia de adaptación a condiciones subóptimas o adversas en los periodos secos que favorezca la producción del grano, advirtiendo que existe un efecto del tipo de árbol sobre las estrategias de adaptación [21].

Los cultivos de cacao asociado con diversas especies de árboles producen condiciones de sombra que permitan un adecuado desarrollo del cultivo y buen desempeño fisiológico de la planta, aprovechando su condición umbrófila (tolerante a la sombra) [22]. Se han implementado sistemas de riego debido a la alta radiación y demanda hídrica, pero por sus altos costos de producción solo se ha implementado en el 0,5\% del área cultivada [23, 24].

De acuerdo a esta situación, los sistemas agroforestales se convierten en una estrategia de adaptación a las condiciones hídricas limitantes especialmente por la alta radiación durante las épocas secas para la producción de cacao. El rol de los SAF para el mantenimiento del estatus hídrico en los árboles de cacao radica en la modificación de las condiciones microclimáticas lo cual incide notablemente en la transpiración.

El potencial hídrico es el trabajo necesario para llevar el agua ligada al nivel del potencial de agua pura $(\mathrm{MPa}=0)$ a una misma temperatura y presión atmosférica [25]. Esta variable influye en el crecimiento celular, la fotosíntesis y la productividad de los cultivos, y es un indicador del estado hídrico del cultivo [26]. El monitoreo del flujo de savia $\left(V_{s}\right)$ y del potencial hídrico del xilema $(\Psi \mathrm{MPa})$ en las especies arbóreas permite estimar la transpiración [27] y entender las respuestas específicas de la planta a la variación de los parámetros ambientales, la disponibilidad de agua y luz [28, 29], para proponer el desarrollo de sistemas de producción estables [30].

Con base en este escenario es necesario desarrollar estudios cuantitativos sobre la respuesta de las plantas de cacao (productividad) al déficit hídrico y maneras de mitigación de los efectos adversos [1,21]. El objetivo del presente estudio fue determinar la influencia de la cobertura arbórea sobre el estatus hídrico (dinámica del flujo de savia y potencial hídrico) en árboles de cacao Theobroma cacao L. en el valle interandino del norte del Huila.

\section{MÉTODO}

La investigación se realizó en el municipio de Campoalegre, Huila, (Colombia) en una finca ubicada a $2^{\circ} 40^{\prime} 30,2^{\prime \prime}$ $\mathrm{N}$ y $75^{\circ} 21^{\prime} 24,6^{\prime \prime} \mathrm{W}, 533 \mathrm{msnm}$, temperatura promedio anual de $25,6^{\circ} \mathrm{C}$, humedad relativa promedio anual de $68 \%$, brillo solar de 1679 horas/año y promedio de 6 horas de sol día; que corresponde a una zona de bosque seco tropical en la región fisiográfica del valle superior del río Magdalena. El régimen de lluvias es bimodal, con una precipitación media anual 1370 mm con dos picos de lluvia: marzo-mayo y octubre a noviembre.

\section{Sistemas de producción cacaoteros}

Se utilizaron cultivos en estado productivo de cacao clonados con el genotipo CCN51 que tenían un marco de siembra $3 \times 3 \mathrm{~m}$, edad promedio de plantación de 8 años, caracterizados por los siguientes sistemas de producción i. a libre exposición solar (SLE) y ii. bajo sistemas agroforestales (SAF). Para los cultivos bajo SAF se experimentaron dos opciones: i. con dominio de frutales (Annona muricata, Morinda citrifolia, Psidium guajava), niveles de radiación transmitida baja $\left(\mathrm{B}_{\mathrm{PAR}}\right)$, dosel del $66 \%$ de apertura, un índice de área foliar promedio de 2,3 y una densidad de árboles frutales de 103 árboles en 0,01 ha ii. con dominio de especies vegetales maderables (Ochroma pyramidale, Cordia alliodora, Cecropia peltata y Guarea guidonia), radiación transmitida media ( $\mathrm{M}_{\mathrm{PAR}}$ ), apertura de dosel de $22 \%$, un índice de área foliar promedio de 3,1 y una densidad de árboles maderables de 12 árboles en 0,01 ha.

Las condiciones de clima y suelo fueron homogéneas para las parcelas de los sistemas de producción evaluados ya que se encontraban en la misma finca, una seguida de la otra. 


\section{Medición del flujo de savia y potencial hídrico}

El flujo de savia $\left(V_{s}\right)$ fue determinado utilizando sensores tipo SFM1 (ICT International, Armidale, Australia), instalados en el tronco principal de tres árboles de cacao en cada parcela ( $n=9)$, a $15 \mathrm{~cm}$ de altura del suelo. Cada sensor SFM1, está conformado por un sistema de recolección de datos (CR10X datalogger + multiplexor AM416, Campbell Scientific, Logan, UT, USA), programado para almacenar los datos cada diez minutos y posteriormente la información semanalmente en el Software ICT HRM (Versión: 2.0.5.4). Los sensores SFM1 se fundamentan en el método de relación de calor (Heat Ratio Method, HRM) [31]. En el tronco de cada árbol de cacao se instalaron dos agujas y una sonda de calor integrada a un microprocesador para determinar la velocidad del flujo de savia. Cada aguja está compuesta por dos termocuplas las cuales reciben el pulso de calor que incrementa la temperatura en el xilema conductor y es proporcional a la velocidad del pulso de calor $\left(V_{s} / \mathrm{cm} / \mathrm{h}\right)[31,32]$.

La $\mathrm{V}_{s}$, se estimó utilizando la ecuación 1.

$$
\mathrm{V}_{\mathrm{S}}=\frac{\mathrm{K}}{\mathrm{X}} \ln \left(\mathrm{T}_{1} / \mathrm{T}_{2}\right) \times 3600 \quad \text { (Ec. 1) }
$$

Donde:

$\mathrm{K}=$ Difusividad térmica en el área del xilema conductor (se asumió un valor por defecto de $0,0025 \mathrm{~cm}^{2} / \mathrm{s}$ ).

$\mathrm{x}=$ Distancia entre la sonda de calor y cada una de las agujas $(\mathrm{cm})$.

$\mathrm{T}=$ Aumento de la temperatura $\left({ }^{\circ} \mathrm{C}\right)$ inicial con respecto a la aguja ubicada en la parte

inferior $\left(T_{1}\right)$ y superior $\left(T_{2}\right)$.

Para determinar el potencial hídrico del xilema ( $\Psi$, Mpa) se utilizaron psicrómetros de tallo PSY (ICT International, Armidale, Australia) instalados a la misma altura del suelo del tronco principal del árbol donde se ubicaron los sensores tipo SFM1. Para el registro y almacenamiento de datos se instaló a cada psicrómetro un "datalogger" Em-50 (Decagon Devices, Pullman, Washington, USA), con frecuencia de registró de diez minutos. Los datos registrados fueron descargados cada semana en el Software PSY1 ICT (Versión: 2.0.5.9). EI భ Mpa se calculó según lo propuesto por [33].

\section{Análisis estadístico}

Los datos obtenidos en dos temporadas contrastantes de precipitación (época de máxima precipitación: del 17 de marzo al 13 de Abril del 2017 y época de mínima precipitación: del 21 de Septiembre al 11 de Octubre de 2017), de flujo de savia y potencial hídrico fueron analizadas ajustando modelos lineales mixtos (MLM) para estudiar el efecto de los factores fijos (i. sistema de producción, ii. Época y iii. Hora). Se incluyeron como efectos aleatorios el día y las repeticiones de los árboles de cacao. Los supuestos de normalidad y homogeneidad de la varianza se evaluaron mediante un análisis residual exploratorio. Las diferencias entre los sistemas de producción se analizaron con la prueba post-hoc de LSD de Fisher con una significación de $\alpha=0,05$. El MLM se realizó utilizando la función Ime en el paquete nIme y las gráficas fueron realiza utilizando el paquete "ggplot2" el software de lenguaje R, versión 3.4.4 (R Development Core Team, 2020), por la interfaz en InfoStat [34]. 


\section{RESULTADOS}

\section{Potencial hídrico}

Al comparar el gradiente del potencial hídrico del xilema conductor $(\Psi)$, este fue mayor en el cultivo a libre exposición solar (SLE) en las dos épocas de monitoreo pero se acentuó más en la época seca cuando muestra valores de -3,08 $\pm 0,1 \mathrm{Mpa}$. En los sistemas agroforestales (SAF) con maderables con medio nivel de radiación transmitida $\left(\mathrm{M}_{\mathrm{PAR}}\right)$ el valor fue intermedio $(-2,29 \pm 0,1 \mathrm{Mpa})$, seguido del SAF con frutales con nivel de radiación transmitida baja $\left(B_{P A R}\right)$ que registró el menor valor de potencial hídrico durante la misma época $(-0,75 \pm 0,1 \mathrm{Mpa})$.

\section{Flujo de savia}

En el flujo de savia $\left(V_{s}\right)$ hay un comportamiento diferente en las dos épocas climáticas en el caso de $S A F$ con frutales $\left(B_{\text {PAR }}\right)$. En la época seca se alcanza el valor extremo entre las 10 y las 15 horas. En el SAF con maderables $\left(M_{P A R}\right)$ las curvas muestran el mismo patrón y en algunos momentos del día se presentan diferencias significativas $(P<0,005)$. En el cultivo a libre exposición solar (SLE) el comportamiento es muy parecido en las dos épocas a lo largo del día.

Se evidencia en la Figura 1, como el flujo de savia en el SAF con frutales $\left(\mathrm{B}_{\mathrm{PAR}}\right)$ durante la época de mínima precipitación tiene el mayor valor $\left(0,5 \mathrm{~L} \mathrm{~h}^{-1}\right)$ a las 12 horas, seguido del SAF con maderables $\left(\mathrm{M}_{\mathrm{PAR}}\right)\left(0,38 \mathrm{~L} \mathrm{~h}^{-1}\right)$ entre las 10 y las 18 horas, frente al cultivo a libre exposición solar (SLE) con un menor valor $\left(0,35 \mathrm{~L} \mathrm{~h}^{-1}\right)$ entre las 12 horas.

Figura 1. Comportamiento del flujo de savia $\left(V_{s}\right)$ y del potencial hídrico $(\Psi, \mathrm{Mpa})$ en árboles de cacao en SLE y SAF ( $B_{P A R}$ con frutales y $M_{P A R}$ con maderables), en máxima y mínima precipitación.

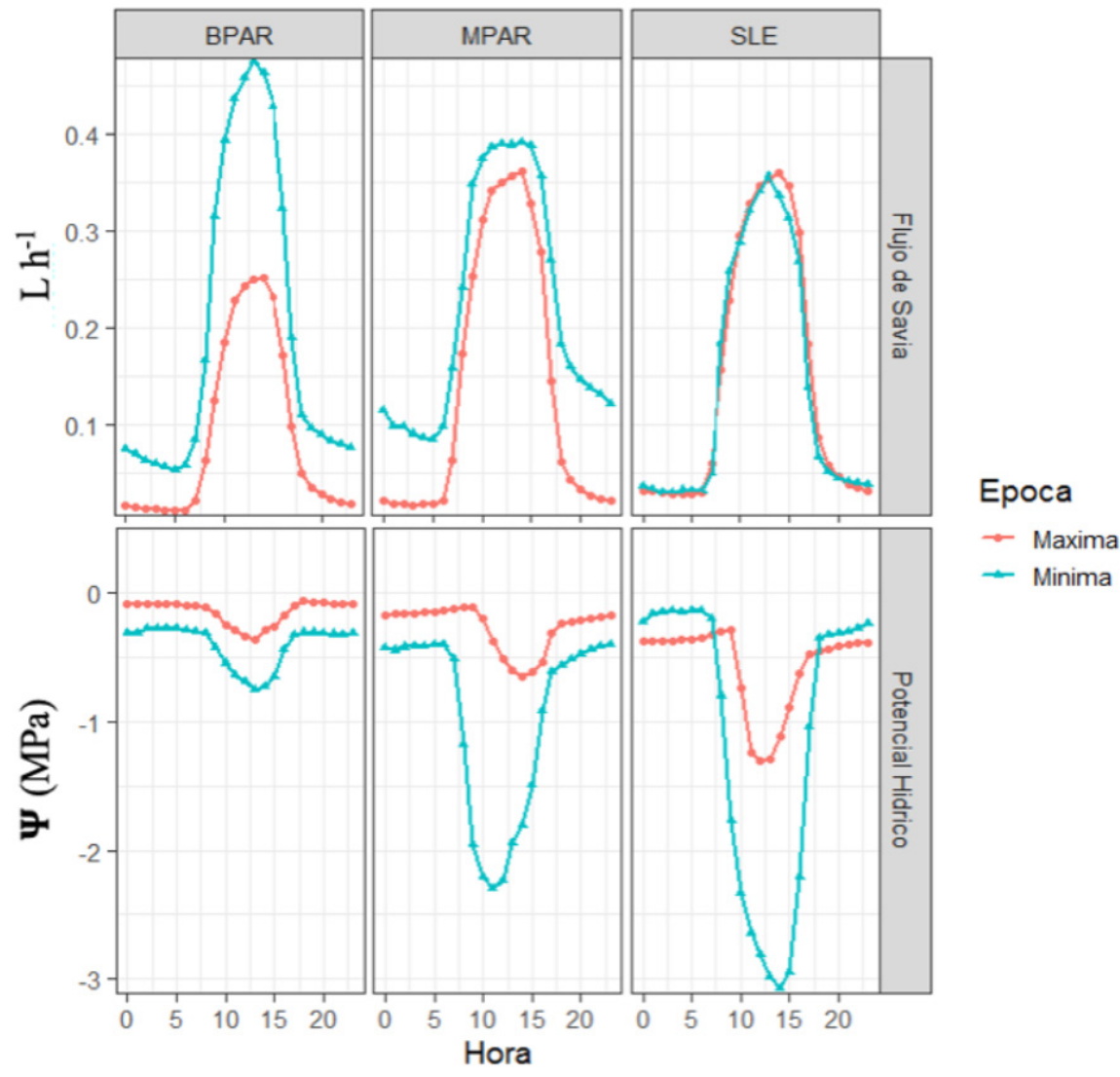




\section{Flujo de savia y potencial hídrico}

Los valores del potencial hídrico $(\Psi)$ se correlacionan de manera negativa con el flujo de savia $\left(V_{s} r=-0,65\right.$ $\mathrm{P}<0,001)$. En la Figura 1, se observa un comportamiento opuesto entre el potencial hídrico ( $\Psi$ ) y el flujo de savia $\left(V_{s}\right)$, entre los sistemas de producción, durante la época seca. En las dos variables $\left(\Psi\right.$ y $\left.V_{s}\right)$ el SAF con frutales $\left(B_{P A R}\right)$ muestra el menor potencial hídrico y mayor flujo de savia, seguido del SAF con maderables $\left(M_{P A R}\right)$ y con los menores valores en el sistema de producción a libre exposición solar (SLE).

En la época de máxima precipitación el mayor flujo de savia se observa en el sistema de producción a libre exposición solar (SLE) y el SAF con maderables $\left(\mathrm{M}_{\mathrm{PAR}}\right)\left(0,36 \pm 0,1 \mathrm{~L} \mathrm{~h}^{-1}\right)$, con un menor valor en el SAF con frutales $\left(B_{\text {PAR }}\right)\left(0,25 \mathrm{~L} \mathrm{~h}^{-1}\right)$, pero el potencial hídrico no tuvo el mismo comportamiento, siendo estadísticamente menor en el SAF ( $\left.B_{\text {PAR }}\right)(-0,3 \pm 0,1 \mathrm{Mpa})$, seguido del $S A F\left(M_{P A R}\right)(-0,6 \pm 0,1 \mathrm{Mpa})$ y el máximo valor en el cultivo a libre exposición solar (SLE) $(-1,5 \pm 0,1 \mathrm{Mpa})(\mathrm{P}<0,01$ Figura 1$)$.

Los resultados descritos sobre el comportamiento del flujo de savia $\left(\mathrm{V}_{s}\right)$ durante la presente investigación, son similares a los descritos por otros estudios [18,22]. Las condiciones que probablemente pueden incidir sobre el comportamiento del estatus hídrico puede ser la variabilidad climática o la variación interanual [35, 36, 37] que ocasiona una alta demanda evaporativa [38] causando el cierre estomático. Estas situaciones han sido descritas en otros estudios específicamente para el cultivo del cacao $[18,38]$ en los cuales mencionan que $V_{\mathrm{s}}$ depende de los incrementos de la radiación y del déficit de vapor de presión. Esto indica el efecto del dosel de sombra sobre procesos de apertura y cierre estomático lo cual se refleja en la dinámica de $\mathrm{V}_{\mathrm{s}}$ [38].

Para el caso del comportamiento del $\Psi$ que se encontró durante la investigación, se puede relacionar con una respuesta fisiológica de la planta a la disponibilidad de agua en el suelo y su distribución en la planta [22] que es limitada por variables atmosféricas [15, 18], específicamente para la época de mínima precipitación. La disminución del agua en la zona de raíces implica ejercer una mayor presión para movilizar el agua desde gradientes diferenciales de presión en el complejo suelo-planta-atmósfera [39, 40, 41, 42].La transpiración excesiva se controla por los estomas; se evitan daños de embolia [43, 44], aunque se reducen la fotosíntesis y el rendimiento (producción) de la planta, causados por la sequía [45]. Se podría considerar que a partir de nuestros resultados probablemente las plantas de cacao tienen un compartimiento isohídrico debido a que cierran sus estomas y se presenta una caída en el potencial hídrico del suelo, es importante advertir que en nuestro estudio el genotipo evaluado es el CCN 51, ya que se ha mencionado que el genotipo puede incidir [46].

Bajo estas condiciones las plantas de cacao en el sistema a libre exposición solar (SLE) durante la época de mínima precipitación, pudieron presentar un estrés hídrico severo (potencial hídrico <-1,76 Mpa) [47], por la sensibilidad de la especie al déficit hídrico [8]. El mecanismo de las plantas ante el déficit hídrico en los tres sistemas de producción analizados reflejo la pérdida de agua de los tejidos del tallo durante la mañana y una recuperación nocturna, exhibiendo una fase opuesta al flujo de savia, estos cambios están estrechamente relacionados con factores climáticos [48, 43, 49, 50, 41] en árboles perennes en el trópico [39].

Los valores de potencial hídrico (-0,4 hasta - $0,75 \mathrm{Mpa}$ ) encontrados en el SAF con frutales $\left(\mathrm{B}_{\mathrm{PAR}}\right)$ durante las dos épocas evaluadas fueron menores a $1 \mathrm{MPa}$ que fisiológicamente indican un estrés hídrico moderado [47]. Esta acción se relaciona con la influencia del dosel sobre el microclima que permite reducir la evapotranspiración comparada con la situación a libre exposición solar [22]. Los resultados hallados comprueban que la cobertura arbórea de los árboles asociados con cacao en Sistemas Agroforestales (SAF), reduce el estrés hídrico, favorece el balance hídrico del cultivo [52], el flujo de savia y en consecuencia mejorar los procesos fotosintéticos y el rendimiento del cultivo $[8,9]$ en épocas de mínima precipitación. 


\section{CONCLUSIONES}

La tendencia del flujo de savia $\left(\mathrm{V}_{s}\right)$ durante el día en los periodos contrastantes de precipitación siguió un patrón similar tanto $B_{P A R}$ y $M_{\text {PAR, }}$ comparado con SLE. Al comparar las épocas se evidencia que el Vs fue mayor en la época seca (mínima precipitación) con una magnitud de $0,5 \mathrm{~L} \mathrm{~h}^{-1}$ bajo el SAF con frutales el cual tenía un bajo nivel de radiación transmitida $\left(B_{\text {PAR }}\right)$. Sin embargo en el sistema a libre exposición solar (SLE) el comportamiento del Vs no fue estadísticamente diferente.

El potencial hídrico $(\Psi)$ mostró los valores más altos $(-0,75,-2,29$ y -3,08 $\mathrm{MPa})$ durante la época seca en los tres arreglos, pero la mayor magnitud se presentó bajo el sistemas a libre exposición solar (SLE), seguida por la condición SAF maderables con radiación transmitida media $\left(M_{P A R}\right)$ y por los SAF con frutales con nivel de radiación transmitida baja $\left(\mathrm{B}_{\mathrm{PAR}}\right)$, donde las variaciones entre las épocas climáticas no son tan extremas.

Los resultados evidencian un efecto positivo de los SAF sobre el estatus hídrico en el cultivo de cacao durante las dos épocas evaluadas, porque mejoran las condiciones microclimáticas que favorecen el balance hídrico del cultivo y reducen el estrés hídrico para que no se limite el flujo de savia, la fotosíntesis y por ende el rendimiento del cultivo. Además de estas los servicio ecosistémicos directos, que convierten los SAF en una estrategia adecuada de adaptación al cambio climático por enriquecer y mantener la biodiversidad asociada a las áreas con plantaciones de cacao.

\section{AGRADECIMIENTOS}

Al Departamento del Huila por apoyar la formación Doctoral, al SENA Regional Huila, a la Universidad de la Amazonía y la Universidad Nacional de Colombia.

\section{REFERENCIAS}

[1] CARR, M.K.V.; LOCKWOOD, G. The water relations and irrigation requirements of cocoa (Theobroma cacao L.) a review. Experimental Agriculture v. 47, n. 04, 2011, p. 653-676. https://doi.org/10.1017/S0014479711000421

[2] SUÁREZ-SALAZAR, JUAN-CARLOS. Comportamiento ecofisiológico de Theobroma cacao L. en diferentes arreglos agroforestales bajo condiciones de la Amazonia Colombiana [Tesis Doctoral en Ciencias Biológicas]. Bogotá (Colombia): Universidad Nacional de Colombia, Facultad de Ciencias, Departamento de Biología, 2018, 147 p.

[3] HEBBAR, K.B.; APSHARA, ELAIN; CHANDRAN, K.P; PRASAD,VARA. Effect of elevated $\mathrm{CO}_{2}$, high temperature, and water deficit on growth, photosynthesis, and whole plant water use efficiency of cocoa (Theobroma cacao L.). International Journal of Biometeorology, v. 64, n. 1, 2020, p. 47-57. https://doi.org/10.1007/s00484-019-01792-0

[4] JANANI, P.; KUMAR, N.; JEGADEESWARI, V. Evaluation of cocoa (Theobroma cacao L.) clones under natural rainfed conditions for drought tolerance. Chemical Science Review and Letters, v. 8, n. 32, 2019, p. 220-225.

[5] JANANI, P.; KUMAR, N.; JEGADEESWARI, V. Dynamics of gas exchange and chlorophyll fluorescence parameters of cocoa genotypes in response to water deficit. Journal of Pharmacognosy and Phytochemistry, v. 8, n. 6, 2019, p. 415-419

[6] KUNIKULLAYA, AMITHA; SURESH, J.; BALAKRISHNAN, S.; KUMAR, M.; JEYAKUMAR, P.; KUMARAVADIVEL, N.; JEGADEESWARI, V. Effect of water stress on photosynthetic parameters of cocoa (Theobroma cacao L.) genotypes. International Journal of Chemical Studies, v. 6, n. 6, 2018, p. 1021-1025.

[7] ACHEAMPONG, K.; DAYMOND, A.J.; ADU-YEBOAH, P.; HADLEY, P. Improving field establishment of cacao (Theobroma cacao) through mulching, irrigation and shading. Experimental Agriculture, v. 56, n. 6 , 2019, p. 898-912.

http://10.1017/S0014479718000479 
[8] NIETHER, WIEBKE; SCHNEIDEWIND, ULF; ARMENGOT, LAURA; ADAMTEY, NOH; SCHNEIDER, MONIKA; GEROLD, GERHARD. Spatial-temporal soil moisture dynamics under different cocoa production systems. Catena v. 158, 2017, p. 340-349. https://doi.org/10.1016/j.catena.2017.07.011

[9] GATEAU-REY, LAURANNE; TANNER, EDMUND; RAPIDEL, BRUNO; MARELLI, JEAN-PHILIPPE; ROYAERT, STEFAN. Climate change could threaten cocoa production: Effects of 2015-16 El Niño-related drought on cocoa agroforests in Bahia, Brazil. PLoS ONE, v. 13, n. 7, 2018, e0200454. https://doi.org/10.1371/journal.pone.0200454

[10] SMITH, SARAH C.; UBILAVA, DAVID. The EI Niño Southern Oscillation and economic growth in the developing World. Global Environmental Change v. 45, 2017. p. 151-164. https://doi.org/10.1016/j.gloenvcha.2017.05.007

[11] LAHIVE, FIONA; HADLEY, PAUL; DAYMOND, ANDREW J. The physiological responses of cacao to the environment and the implications for climate change resilience. A review. Agronomy for Sustainable Development, v. 39, n. 5, 2019. https://doi.org/10.1007/s13593-018-0552-0

[12] BECA, KERSTIN; KREYLING, JUERGEN; BEIERKUHNLEIN, CARL; JENTSCH, ANKE. Importance of Seasonality for the Response of a Mesic Temperate Grassland to Increased Precipitation Variability and Warming. Ecosystems, v.20, 2017, p. 1454-1467. https://doi.org/10.1007/s10021-017-0122-3

[13] MEDINA, V.; LALIBERTE, B. A review of research on the effects of drought and temperature stress and increased $\mathrm{CO}_{2}$ on Theobroma cacao $\mathrm{L}$, and the role of genetic diversity to address climate change. San José (Costa Rica): Bioversity International, v. 51, 2017, p. 1-59.

[14] DE ALMEIDA, JENNY; TEZARA, WILMER; HERRERA, ANA. Respuestas fisiológicas a la sequía y el déficit de agua experimental y el anegamiento de cuatro clones de cacao (Theobroma cacao $\mathrm{L}$ ) seleccionados para el cultivo en Venezuela. Agricultural Water Management v. 171, 2016, p. 80-88. https://doi.org/10.1016/j.agwat.2016.03.012

[15] MOSER, G.; LEUSCHNER, C.; HERTEL, D.; HÖLSCHER, D.; KÖHLER, M.; LEITNER, D.; MICHALZIK, B.; PRIHASTANTI, E.; TJITROSEMITO, S.; SCHWENDENMANN, L. Response of cocoa trees (Theobroma cacao) to a 13-month desiccation period in Sulawesi, Indonesia. Agroforestry Systems, v. 79, n. 2, 2010, p. 171-187. https://doi.org/10.1007/s10457-010-9303-1

[16] KOTOWSKA, MARTYNA M.; HERTEL, DIETRICH; RAJAB, YASMIN-ABOUT; BARUS, HENRY; SCHULDT, BERNHARD. Patterns in hydraulic architecture from roots to branches in six tropical tree species from cacao agroforestry and their relation to wood density and stem growth. Frontiers in Plant Science, v. 6, 2015, p. 1-16. https://doi.org/10.3389/fpls.2015.00191

[17] QUINTANA-FUENTES, LUCAS-FERNANDO; GARCÍA-JEREZ, ALBERTO; MORENO-MARTÍNEZ, EDITH. Perfil sensorial de cuatro modelos de siembra de cacao en Colombia. Entramado, v. 14, n. 2, 2018, p. 256-268. http://dx.doi.org/10.18041/1900-3803/entramado.2.4756

[18] KÖHLER, MICHAEL; HANF, ANDREA; BARUS, HENRY; HÖLSCHER, DIRK. Cacao trees under different shade tree shelter: effects on water use. Agroforestry systems, v. 88, n. 1, 2014, p. 63-73. https://doi.org/10.1007/s10457-013-9656-3

[19] NIETHER, WIEBKE; ARMENGOT, LAURA; ANDRES, CRISTIANO; SCHNEIDER, MONIKA; GEROLD, GERHARD. Shade trees and tree pruning alter throughfall and microclimate in cocoa (Theobroma cacao L.) production systems. Annals of forest science, v. 75, n. 38, 2018, p. 1-16. https://doi.org/10.1007/s13595-018-0723-9

[20] BORDEN, KIRA A.; ANGLAAERE, LUKE; OWUSU, SANDRA; MARTIN, ADAM; BUCHANAN, SERRA W.; ADDO-DANSO, SHALOM D.; ISAAC, MARNEYE. Soil texture moderates root functional traits in agroforestry systems across a climatic gradient. Agriculture, Ecosystems \& Environment, v. 295, 2020, p. 1-5. https://doi.org/10.1016/j.agee.2020.106915 
[21] ABDULAI, ISSAKA; VAAST, PILIPPE; HOFFMANN, MUNIR P.; ASARE, RICHARD; JASSOGNE, LAURENCE; VAN-ASTEN, PIEN; RÖTTER, REIMUND; GRAEFE, SOPHIE. Cocoa agroforestry is less resilient to suboptimal and extreme climate than cocoa in full sun. Global change biology, v. 24, n. 1, 2018, p. 273-286. https://doi.org/10.1111/gcb.13885

[22] JIMÉNEZ-PÉREZ, ALFREDO; CACH-PÉREZ, MANUEL; VALDEZ-HERNÁNDEZ, MIRNA; DE LA ROSA-MANZANO, EDILIA. Effect of canopy management in the water status of cacao (Theobroma cacao) and the microclimate within the crop area. Botanical Sciences, v. 97, n. 4, 2019, p. 701-710. https://doi.org/10.17129/botsci.2256

[23] MENESES-BUITRAGO, DIEGO-HERNÁN; BOLAÑOS-BENAVIDES, MARTHA-MARINA; GÓMEZ-GIL, LUIS-FERNANDO; RAMOS-ZAMBRANO, HOUSSEMAN-STEVEN. Evaluation of irrigation and pruning on the phenology and yield of Theobroma cacao L. Agronomía Mesoamericana, v. 30, n. 3, 2019, p. 681-693. http://10.15517/am.v30i3.36307

[24] BUNN, C.; FERNANDEZ-KOLB, P.; ASARE, R.; LUNDY, M. Climate Smart Cocoa in Ghana Towards climate resilient production at scale. CCAFS Info Note. CGIAR Research Program on Climate Change, Agriculture and Food Security (CCAFS) [online]. 2019. Disponible: https://cgspace.cgiar.org/handle/10568/103770 [citado 22 de Mayo de 2020].

[25] MELGAREJO, LUZ-MARINA; HERNÁNDEZ, SOLEDAD; BARRERA, JAIME; SOLARTE, MARIA-ELENA.; SUAREZ, DIEGO.; PEREZ, LAURA-VICTORIA.; ROJAS, YADIRA-ANDREA; CRUZ-AGUILAR, MARISOL; MORENO-ALVAREZ, LEONARDO-GUILLERMO; CRESPO, SANDRA; PÉREZ, WALTER. Experimentos en fisiología vegetal. Bogotá (Colombia): Universidad Nacional de Colombia, 2010, p. 137-167. Disponible:http://bdigital.unal.edu.co/8545/2/02_Preliminares.pdf

[26] FERREYRA, RAUL; SELLES, GABRIE L.; MALDONADO, PATRICIO; CELEDÓN, JOSÉ; GIL, P. Efecto del Clima, de las Características de la Hoja y de la Metodología de Medición en el Potencial Hídrico Xilemático en Palto (Persea americana Mill.). Agricultura Técnica, v. 67, n. 2, 2007, p. 182-188.

[27] CASSIANI, G.; BOAGA, J.; VANELLA, D.; PERRI, M.T.; CONSOLI, S. Monitoring and modelling of soil-plant interactions: the joint use of ERT, sap flow and eddy covariance data to characterize the volume of an orange tree root zone. Hydrology and Earth System Sciences, v. 19, n. 5, 2015, p. 2213-2225.

[28] MINER, GRACE-LLOYD; HAM, JAY M.; KLUITENBERG, GERARD L. A heat-pulse method for measuring sap flow in corn and sunflower using 3D-printed sensor bodies and low-cost electronics. Agricultural and Forest Meteorology, v. 246, 2017, p. 86-97.

https://doi.org/10.1016/j.agrformet.2017.06.012

[29] EPILA, JAKIE; MAES, WOUTER H.; VERBEECK, HANS; VAN-CAMP, JANNE; LAMORIS-OKULLO, JOHN-BOSCO; STEPPE, KATHY. Plant measurements on African tropical Maesopsis eminii seedlings contradict pioneering water use behaviour. Environmental and experimental botany, v. 1, n. 35, 2017, p. 27-37. https://doi.org/10.1016/j.envexpbot.2016.12.006

[30] SHUAI, FU; SUN, LI; LUO, YI. Canopy conductance and stand transpiration of Populus simonii Carr in response to soil and atmospheric water deficits in farmland shelterbelt, Northwest China. Agroforestry Systems, v. 91, n. 6, 2016, p. 1165-1180. https://doi.org/10.1007/s10457-016-0002-4

[31] BURGESS, STHEPE; ADAMS, MARK; TURNER, NEIL C.; BEVERLY, CRAIG R.; ONG, CHIN K.; KHAN, AHMED A.; BLEBY, TIM M. An improved heat pulse method to measure low and reverse rates of sap flow in woody plants. Tree Physiology, v. 21, 2001, p. 589-598.

https://doi.org/10.1093/treephys/21.9.589

[32] BURGESS, STHEPEN; ADAMS, MARK; TURNER, NEIL C.; ONG, CHIN K. The redistribution soil water by tree root systems. Oecologia v. 15, 1998, p. 306-311. https://doi.org/10.1007/s004420050521

[33] DIXON, MIKE; DOWNEY, ALEC. PSY1 Stem Psychrometer Manual. ICT International [online]. 2013. Disponible: http://www.ictinternational.com/content/uploads/2014/03/PSY1-manual-ver-4.7.pdf [citado 2 de Mayo de 2020]. 
[34] DI RIENZO, J.A.; CASANOVES, F.; BALZARINI, M.G.; GONZALEZ, L.; TABLADA, M.; ROBLEDO, C.W. InfoStat versión 2017. Grupo InfoStat, FCA, Universidad Nacional de Córdoba, Argentina. [online]. 2017. Disponible: http://www.infostat.com.ar [citado 8 de mayo de 2020].

[35] ZHAO, CHUNYAN; SI, JIAN-HUA; FENG, QI; YU, TENGFEY; LI, PEIDU. Comparative study of daytime and nighttime sap flow of Populus euphratica. Plant Growth Regulation, v. 82, n. 2, 2017, p. 353-362. https://doi.org/10.1007/s10725-017-0263-6

[36] XIA, J.B.; ZHAO, Z.G.; SUN, J.K.; LIU, J.T.; ZHAO, Y.Y. Response of stem sap flow and leaf photosynthesis in Tamarix chinensis to soil moisture in the Yellow River Delta, China. Photosynthetica, v. 55, 2017, p. 368-377. https://doi.org/10.1007/s11099-016-0651-6

[37] QIU-YUE, HE; MEI-JIE, YAN; MIYAZAWA, YOSHIYUKI; QIU-WEN, CHEN; RAN-RAN, CHENG; KYOICHI, OTSUKI; NORIKAZU, YAMANAKA.; SHENG, DU. Sap flow changes and climatic responses over multiple-year treatment of rainfall exclusion in a sub-humid black locust plantation. Forest Ecology and Management, v. 457, 117730, 2020. https://doi.org/10.1016/j.foreco.2019.117730

[38] KÖHLER, MICHAEL; DIERICK, DIEGO; SCHWENDENMANN, LUITGARD; HÖLSCHER, DIRK. Water use characteristics of cacao and Gliricidia trees in an agroforest in Central Sulawesi, Indonesia. Ecohydrology: Ecosystems, Land and Water Process Interactions, Ecohydrogeomorphology, v. 2, n. 4, 2009, p. 520-529. https://doi.org/10.1002/eco.67

[39] LUO, ZINDONG; GUAN, HUADE; ZHANG, XINPING; ZHANG, CICHENG; LIU, NA; LI, GUANG. Responses of plant water use to a severe summer drought for two subtropical tree species in the central southern China. Journal of Hydrology: Regional Studies, v. 8, 2016, p. 1-9. https://doi.org/10.1016/j.ejrh.2016.08.001

[40] CARMINATI, ANDREA; JAVAUX, MATHIEU. Soil Rather Than Xylem Vulnerability Controls Stomatal Response to Drought. Trends in Plant Science, TRPLSC 1962, 2020, p.1-13. https://doi.org/10.1016/j.tplants.2020.04.003

[41] RODRIGUEZ-DOMINGUEZ, CELIA M.; BRODRIBB, THIMOTY J. Declining root water transport drives stomatal closure in olive under moderate water stress. New Phytologist, v. 225, n. 1, 2020, p. 126-134. https://doi.org/10.1111/nph.16177

[42] HAN, MIN; ZHANG, HUIHUI; DEJONGE, KENDALL C.; COMAS, LUIS H.; GLEASON, SEAN. Comparison of three crop water stress index models with sap flow measurements in maize. Agricultural Water Management, v. 203, 2018, p 366-375. https://doi.org/10.1016/j.agwat.2018.02.030

[43] FENG, YU; CUI, NINGBO; DU, TAISHENG; GONG, DAOZHI; HU, XIAOTAO; ZHAO, LU. Response of sap flux and evapotranspiration to deficit irrigation of greenhouse pear-jujube trees in semi-arid northwest China. Agricultural water management, v. 194, 2017, p. 1-12. https://doi.org/10.1016/j.agwat.2017.08.019

44] DE OLIVEIRA, PRISCILA; SILVA-PEREIRA, LIDIANE; COSTA-SILVA, DEMIRA; DE SOUZA-JÚNIOR, JÓSE-OLIMPIO; GALVES-LAVIOLA, BRUNO; PINTO-GOMES, FABIO. Hydraulic conductivity in stem of young plants of Jatropha curcas L. cultivated under irrigated or water deficit conditions. Industrial Crops and Products, v. 116, 2018, p. 15-23.

https://doi.org/10.1016/j.indcrop.2017.12.066

[45] LI, SHAN; JANSEN, STEVEN. The root cambium ultrastructure during drought stress in Corylus avellana. IAWA journal, v. 38, n. 1, 2017, p. 67-80. https://doi.org/10.1163/22941932-20170157

[46] HUGALDE, INES-PILAR; VILA, HERNAN-FELIX. Comportamiento isohídrico o anisohídrico en vides.... ¿Una controversia sin fin? [online]. 2014. Disponible:http://ria.inta.gob.ar/sites/default/files/trabajosenprensa/art.7hugalde-comportamiento.pdf [citado 30 de Mayo de 2020].

[47] DENG, XIMIN; JOLY, ROBERT; HAHN, DANIEL. The influence of plant water deficit on distribution of 14C-labelled assimilates in cacao seedlings. Annals of botany v. 66, 1990, p. 211-217.

https://doi.org/10.1093/oxfordjournals.aob.a088017 
[48] SCOFFONI, CHRISTINE; ALBUQUERQUE, CAETANO; BRODERSEN, CRAIG R.; TOWNES, SHATARA V.; JOHN, GRACE P.; COCHARD, HERVÉ; BUCKLEY, THOMAS N.; SACK, LAWREN. Leaf vein xylem conduit diameter influences susceptibility to embolism and hydraulic decline. New Phytologist, v. 213, n. 3, 2017 , p. 1076-1092. https://doi.org/10.1111/nph.14256

[49] WU, YUANZHI; ZHANG, YONGKUN; AN, JUAN; LIU, QIANJIN; LANG, YING. Sap flow of black locust in response to environmental factors in two soils developed from different parent materials in the lithoid mountainous area of North China. Trees, v. 32, n. 3, 2018, p. 675-688. https://doi.org/10.1007/s00468-018-1663-6

[50] ZHOU, H.; SUN, Y.; SHAN, G.; GRANTZ, D.A.; CHENG, Q.; LAMMERS, P.S.; CHEN, B. In situ measurement of stem water content and diurnal storage of an apricot tree with a high frequency inner fringing dielectric sensor. Agricultural and Forest Meteorology, v. 250, 2018, p. 35-46. https://doi.org/10.1016/j.agrformet.2017.12.002

[51] DAMM, A.; PAUL-LIMOGES, E.; HAGHIGHI, E.; SIMMER, C.; MORSDORF, F.; SCHNEIDER, F.D.; RASCHER, U. Remote sensing of plant-water relations: An overview and future perspectives. Journal of plant physiology, v. 227, 2018, p. 3-19. https://doi.org/10.1016/j.jplph.2018.04.012

[52] DA SILVA-RIBEIRO, GABRIELLA; BBUD-RIGHI, CIRO. Canopy architecture of an agroforestry system: initial evaluation of a waveform system. Agroforest Syst, v. 94, 2020, p. 487-498. https://doi.org/10.1007/s10457-019-00415-2 